\title{
Teores de Água no Solo e EFicácia do Herbicida Glyphosate no CONTROLE DE Euphorbia heterophylla
}

\author{
Soil Water Contents and Glyphosate Efficacy in Controlling Euphorbia heterophylla
}

\author{
ZANATTA, J.F. ${ }^{2}$, PROCÓPIO, S.O. ${ }^{3}$, MANICA, R. ${ }^{4}$, PAULETTO, E.A. ${ }^{5}$, CARGNELUTTI FILHO, A. ${ }^{6}$, \\ VARGAS, L. ${ }^{7}$, SGANZERLA, D.C. ${ }^{4}$, ROSENTHAL, M.D.A. ${ }^{8}$ e PINTO, J.J.O. ${ }^{9}$
}

\begin{abstract}
RESUMO - Um experimento em casa de vegetação foi realizado com os objetivos de avaliar a eficácia do herbicida glyphosate no controle de Euphorbia heterophylla se desenvolvendo em solo com diferentes teores de água e determinar qual o menor teor de água do solo que não prejudica a ação desse herbicida no controle dessa planta daninha. $O$ delineamento utilizado foi o de blocos casualizados em esquema fatorial $6 \times 5$, sendo seis intervalos entre a última irrigação e a aplicação do herbicida $(0,6,12,24,48$ e 72 horas) e cinco doses de glyphosate $\left(0,180,360,720\right.$ e $\left.1.080 \mathrm{~g} \mathrm{ha}^{-1}\right)$. Quando as plantas atingiram estádio de três pares de folhas, foram aplicados $10 \mathrm{~mm}$ de chuva simulada, conforme tratamento previsto. Ao término do período de simulação de chuva, aplicou-se o herbicida com pulverizador costal pressurizado com $\mathrm{CO}_{2}$, utilizando-se volume de calda de $120 \mathrm{~L} \mathrm{ha}^{-1}$. Aos 7, 20, 34 e 41 dias após aplicação (DAA), foram avaliados o controle (por escala visual) e, aos 41 DAA, a massa seca de raízes e da parte aérea. Após análise dos dados, verificou-se que a partir da dose de $720 \mathrm{~g} \mathrm{ha}^{-1}$ de glyphosate obteve-se controle satisfatório de E. heterophylla, independentemente do intervalo entre a última irrigação e a aplicação do herbicida. Pulverizações de $360 \mathrm{~g}^{\text {ha }}{ }^{-1}$ de glyphosate a intervalos menores que 48 horas entre a última irrigação e sua aplicação e em solo com teor de água maior que $0,09 \mathrm{~cm}^{3} \mathrm{~cm}^{-3}$ não prejudicaram a eficácia do herbicida. A aplicação de $180 \mathrm{~g}^{\text {ha }}{ }^{-1}$ de glyphosate a intervalos menores que 12 horas entre a última irrigação e sua aplicação e em solo com teor de água superior a $0,14 \mathrm{~cm}^{3} \mathrm{~cm}^{-3}$ não afetou a eficácia do herbicida.
\end{abstract}

Palavras-chave: inibidores de EPSPs, déficit hídrico, planta daninha.

\begin{abstract}
A greenhouse experiment was carried out to evaluate the efficacy of glyphosate in controlling Euphorbia heterophylla growing under different soil moisture levels and to determine the lowest soil moisture level failing to prevent herbicide effectiveness in controlling this weed. The experiments were arranged in a randomized block design in a $6 \times 5$ factorial scheme, corresponding to six intervals between the last irrigation and herbicide application (0, 6, 12, 24, 48 and $72 \mathrm{hrs}$ ) and five glyphosate doses(0, 180, 360, 720, and $\left.1.080 \mathrm{~g} \mathrm{ha}^{-1}\right)$. When the plants achieved the 3-leaf pair stage, $10 \mathrm{~mm}$ of simulated rain were applied. At the end of the rain simulation period, the herbicide was applied using a pressurized backpack sprayer with $\mathrm{CO}_{2}$, using $120 \mathrm{~L} \mathrm{ha}$ of spray volume. On days 7, 20, 34 and 41 after application, the control was evaluated by using a visual scale as well plant height (cm) and on day 41, the dry weight of both the root and aerial part was assessed. Satisfactory control levels of E. heterophylla were verified at a dose of $720 \mathrm{~g} \mathrm{ha}^{-1}$ glyphosate, regardless of the time interval between the last irrigation and herbicide application. Application of $360 \mathrm{~g} \mathrm{ha}^{1}$ of glyphosate at time intervals shorter
\end{abstract}

Recebido para publicação em 15.1.2007 e na forma revisada em 25.10.2007.

2 Mestrando em Fitossanidade, UFPel, Pelotas-RS, 96.010-900; ${ }^{3}$ Pesquisador, Embrapa Tabuleiros Costeiros, Aracaju-SE, 49.025-040, <procopio@cpatc.embrapa.br>; ${ }^{4}$ Acadêmico de Engenharia Agronômica, UFPel; ${ }^{5}$ Professor, Dep. de Solos, UFPel; ${ }^{6}$ Professor do Dep. de Estatística da UFRGS, 90.040-060, Porto Alegre-RS; ${ }^{7}$ Pesquisador, Embrapa Trigo, Passo Fundo-RS, 99.001-970; ${ }^{8}$ Eng $^{\mathrm{e}}$.'Agr ${ }^{\mathrm{e}}$., Dep. de Fitossanidade, UFPel; ${ }^{9}$ Professor, Dep. de Fitossanidade, UFPel. 
than $48 \mathrm{hrs}$, between the last irrigation and its application and in soils presenting water contents higher than $0.09 \mathrm{~cm}^{3} \mathrm{~cm}^{-3}$, did notprevent herbicide efficacy. Application of $180 \mathrm{~g} \mathrm{ha}^{-1}$ of glyphosate at intervals shorter than $12 \mathrm{hrs}$ between the lastirrigation and its application and in soils presenting water contents higher than $0.14 \mathrm{~cm}^{3} \mathrm{~cm}^{-3}$ did not affect herbicide efficacy.

Keywords: EPSPs inhibitors, water deficit, weed.

\section{INTRODUÇÃO}

A espécie Euphorbia heterophylla, conhecida como leiteira ou amendoim-bravo, é uma planta daninha alógama, cujo centro de origem está compreendido em regiões tropicais e subtropicais do continente americano, estando amplamente distribuída num cinturão ao norte e ao sul da linha do Equador, avançando para as regiões subtropicais (Kissmann \& Groth, 1999). Essa espécie está disseminada no centro-sul do Brasil, constatando-se sua presença em $74 \%$ das áreas de soja do planalto do Estado do Rio Grande do Sul (Vidal \& Winkler, 2002). É considerada uma planta invasora de grande importância, que pode causar consideráveis perdas na produtividade, como tem sido verificado nas culturas de amendoim e soja (Moore et al., 1990; Bridges et al., 1992; Willard \& Griffin, 1993).

O uso de herbicidas no manejo de plantas daninhas tem se constituído em uma prática cada vez mais importante na agricultura. No entanto, para a máxima eficiência é necessário o equilíbrio entre muitos fatores ambientais (Santos et al., 2004).

O glyphosate é pertencente ao grupo dos inibidores da enzima EPSPs e contém o N(fosfonometil) glicina como ingrediente ativo (Rodrigues \& Almeida, 1998). É um herbicida sistêmico, não-seletivo, altamente solúvel em água, e seu mecanismo de ação baseia-se na interrupção da rota do ácido chiquímico, responsável pela produção dos aminoácidos aromáticos fenilalanina, tirosina e triptofano, essenciais para a sintese de proteínas e divisão celular nas regiões meristemáticas da planta (Hess, 1994).

Partindo de uma determinada dose aspergida, somente uma fração é retida pelas folhas, absorvida, translocada e chega aos plastídeos, onde está localizada a rota metabólica onde esse composto atua. Cada um desses processos é influenciado por variáveis físicas, ambientais e fisiológicas (Feng et al., 2003). Por exemplo, a retenção foliar pode ser afetada pela interceptação da pulverização (efeito guarda -chuva) e pelo escorrimento das gotículas (De Ruiter et al., 1990), a qual pode ser afetada pela morfologia da planta (ex.: orientação da folha e estrutura e composição da cutícula). Condições ambientais (temperatura e umidade), concentração do surfatante e tamanho da gota (Caseley \& Coupland, 1985; Feng et al., 2003) e o teor de umidade do solo (Victoria Filho, 1985; Kogan \& Bayer, 1996) também influenciam a absorção e a translocação do herbicida.

No caso da planta daninha Euphorbia heterophylla, Ferreira et al. (2003) relataram como possiveis principais barreiras foliares à penetração de agroquímicos o alto teor de cera epicuticular, a elevada densidade de laticíferos e a grande espessura da cutícula da face adaxial. A baixa eficácia de controle do glyphosate em Brunnichia ovata foi relatada ser devido à natureza hidrofóbica da cera epicuticular das folhas dessa espécie (Chachalis et al., 2001). Mendonça (2000) e Monqueiro et al. (2004) observaram que Commelina benghalensis apresentou ceras epicuticulares com maior porcentagem de compostos apolares, o que pode prejudicar a absorção de herbicidas com baixo kow, como o glyphosate.

A umidade relativa e a temperatura têm sido relatadas por influenciarem a absorção e a translocação de herbicidas aplicados em pósemergência. O herbicida chlorsulfurom foi mais tóxico para Setaria viridis e Kochia scoparia quando estas cresciam em 95 a 100\% de umidade relativa do que quando em 45 a 50\% (Nalewaja \& Woznica, 1985). Plantas de S. viridis que cresciam a uma temperatura durante o dia/ noite de $22 / 17^{\circ} \mathrm{C}$ sobreviveram ao herbicida glufosinate na dose de $100 \mathrm{~g} \mathrm{ha}^{-1}$ a $40 \%$ de umidade relativa, mas foram controladas em 
umidade de 95\% (Anderson et al., 1993). Sharma $\&$ Singh (2001) verificaram que a absorção e a translocação de ${ }^{14} \mathrm{C}$-glyphosate em Desmodium tortuosum foram maiores a $22{ }^{\circ} \mathrm{C}$ e $95 \%$ de umidade relativa do que a 16 e $45^{\circ} \mathrm{C}$ ou 35 e $70 \%$ de umidade.

Outro fator que influencia a eficiência de herbicidas é o teor de umidade do solo. Resultados de campo e casa de vegetação indicam que herbicidas aplicados sobre a folhagem durante períodos de seca não são tão efetivos quanto aqueles aplicados quando a umidade do solo está adequada (Boydston, 1990; Peregoy et al., 1990). Segundo Basler et al. (1961) e Levene \& Owen (1995), a baixa eficiência dos herbicidas aplicados durante períodos de déficit hídrico se deve, principalmente, à redução na absorção desses compostos.

Levene \& Owen (1995) demonstraram que, quando plantas de Xanthium strumarium e Abutilon theophrasti estão crescendo em condições de estresse hídrico, elas produzem mais cera epicuticular do que em condições nãoestressantes. Essa maior produção de cera é relatada como a principal barreira à penetração de herbicidas. Freqüentemente, a absorção de herbicidas é reduzida em folhas de plantas em estresse hídrico, pois as folhas perdem a turgescência e enrolam-se, reduzindo a área superficial de interceptação e/ou retenção dos herbicidas (Kidder \& Behrens, 1988, 1991). Moosavi-nia \& Dore (1979) verificaram que em solos com baixo teor de umidade a eficiência de glyphosate no controle de Imperata cylindrica e Cyperus rotundus foi reduzida.
Objetivou-se neste trabalho avaliar a eficácia do herbicida glyphosate no controle de Euphorbia heterophylla se desenvolvendo em solo com diferentes teores de água, determinando qual o menor teor de água no solo que não prejudica a ação desse herbicida no controle dessa planta.

\section{MATERIAL E MÉTODOS}

O trabalho foi desenvolvido em casa de vegetação localizada no campus da Universidade Federal de Pelotas (UFPel), em Capão do Leão, RS, no ano de 2004/2005.

Vasos com capacidade para $1 \mathrm{dm}^{3}$ foram preenchidos com terra de um solo Planossolo Hidromórfico Eutrófico, anteriormente analisado química e fisicamente (Tabela 1). A calagem e adubação da terra foram baseadas na análise físico-química e de acordo com as recomendações técnicas de adubação e calagem para a cultura da soja (Rolas, 2004). Utilizouse a espécie de planta daninha leiteira (Euphorbia heterophylla). Os tratamentos foram compostos pela combinação de cinco doses do herbicida glyphosate $[(0,180,360,720$, e $1.080 \mathrm{~g} \mathrm{ha}^{-1}$ de equivalente ácido (e.a.)] e de seis intervalos entre a última irrigação das plantas e a aplicação do herbicida $(0,6,12$, 24,48 e 72 horas). O delineamento experimental utilizado foi o de blocos casualizados em esquema fatorial $5 \times 6$, com quatro repetições.

Preenchidos os vasos, realizou-se a semeadura da planta daninha. Após a emergência das plantas foi realizado desbaste, deixando-

Tabela 1 - Análise físico-química do solo Planossolo Hidromórfico Eutrófico presente na área experimental. Capão do Leão, RS, 2004

\begin{tabular}{|c|c|c|c|c|c|c|c|c|}
\hline \multicolumn{9}{|c|}{ Análise granulométrica (\%) } \\
\hline Argila & \multicolumn{2}{|c|}{ Silte } & \multicolumn{2}{|c|}{ Areia } & \multicolumn{4}{|c|}{ Classificação textural } \\
\hline 20,28 & \multicolumn{2}{|c|}{31,79} & \multicolumn{2}{|c|}{47,93} & \multicolumn{4}{|c|}{ Franca } \\
\hline \multicolumn{9}{|c|}{ Análise química } \\
\hline \multirow[t]{2}{*}{$\mathrm{pH}$} & $\begin{array}{l}\text { IND } \\
\text { SMP }\end{array}$ & $\mathrm{MO}$ & $\mathrm{P}$ & $\mathrm{K}$ & $\mathrm{Na}$ & $\mathrm{Al}$ & $\mathrm{Ca}$ & $\mathrm{Mg}$ \\
\hline & & $(\%)$ & \multicolumn{3}{|c|}{------------- $\mathrm{mg} \mathrm{kg}^{-1}$} & \multicolumn{3}{|c|}{---------- meq 100 mL $^{-1}$---------- } \\
\hline 4,7 & 5,5 & 2,4 & 8,2 & 80,0 & 13,0 & 1,6 & 2,6 & 1,0 \\
\hline
\end{tabular}

Análises realizadas nos Laboratórios de Análises Físicas e Químicas de Solo do Departamento de Solos da UFPel, segundo a metodologia descrita pela Empresa Brasileira de Pesquisa Agropecuária-Embrapa (1997). 
se quatro plantas por vaso. Nesse mesmo período, coletaram-se três amostras do solo, de três vasos ao acaso, em anéis inox de aproximadamente $50 \mathrm{~cm}^{3}$, para determinação da curva de retenção de água, segundo metodologia descrita por Klute (1986), utilizando o modelo de ajuste proposto por Genuchten (1980). Quando as plantas atingiram o estádio de três pares de folhas, foi realizada a aplicação de $10 \mathrm{~mm}$ de água (simulação de chuva), conforme a época prevista no tratamento, utilizando pulverizador pressurizado com $\mathrm{CO}_{2}$ munido de uma barra com quatro pontas de pulverização do tipo cone cheio 110-02. Após simulação de chuva, as plantas não mais receberam água até o momento da aplicação do herbicida. Posteriormente, à espera dos intervalos, o herbicida foi aplicado em pós-emergência em todos os tratamentos, utilizando-se pulverizador pressurizado com $\mathrm{CO}_{2}$, com barra com uma ponta de pulverização do tipo leque 110-015, resultando em volume de calda equivalente a $120 \mathrm{~L} \mathrm{ha}^{-1}$.

Desde o início do período de simulação de chuva até o momento da aplicação foram monitoradas, durante o dia e à noite, a temperatura e umidade relativa do ar, utilizando um termoigrômetro digital (Figura 1).

Ao término dos períodos de simulação de chuva e antes da aplicação do herbicida, foi realizada a coleta de cinco amostras de solo de diferentes vasos em cada intervalo, para determinação da umidade pelo método da estu-

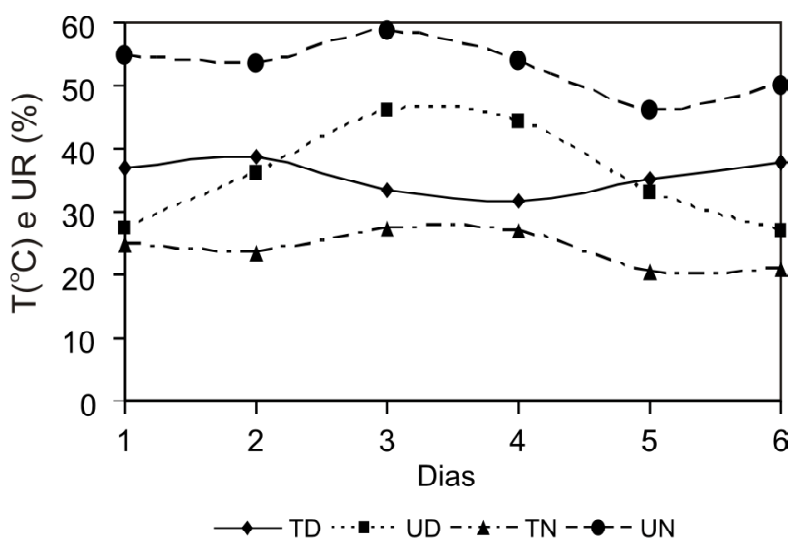

Figura 1 - Média da temperatura e umidade relativa do ar durante o dia (TD, UD) e à noite (TN, UN) no interior da casa de vegetação, desde o período de início da irrigação até a aplicação do herbicida. fa (Embrapa, 1997). A partir dos teores de água obtidos em cada intervalo, determinou-se o seu correspondente potencial mátrico, explicitando $\psi$ da equação de Genuchten, conforme a seguir:

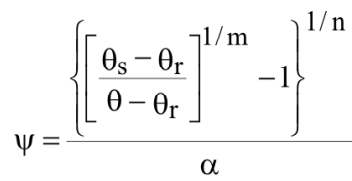

em que:

$\theta=$ umidade volumétrica saturada $\left(\mathrm{cm}^{3} \mathrm{~cm}^{-3}\right)$;

$\theta_{\mathrm{r}}=$ parâmetro de ajuste $\left(\mathrm{cm}^{3} \mathrm{~cm}^{-3}\right)$;

$\psi=$ potencial mátrico $(\mathrm{em} \mathrm{cm})$ de coluna de água; e

$\alpha, \mathrm{m}$ e $\mathrm{n}=$ parâmetros de ajuste referentes às características do solo, determinados de forma empírica.

Vinte e quatro horas após a aplicação do herbicida, todos os vasos foram mantidos sob irrigação, sendo realizados três turnos de rega ao dia.

Aos 7, 20, 34 e 41 dias após aplicação do herbicida (DAA) avaliou-se o controle visualmente, por meio de escala percentual variando de 0 a $100 \%$, indicando ausência de controle e morte da planta, respectivamente. Aos 41 dias após aplicação (DAA), foram quantificadas também a massa seca das raízes e a da parte aérea das plantas, após a coleta e secagem em estufa a $70 \pm 0,2{ }^{\circ} \mathrm{C}$ por $72 \mathrm{~h}$.

Depois da coleta e tabulação dos dados, estes foram submetidos à análise de variância. A análise dos efeitos significativos foi realizada por análise de regressão, sendo os coeficientes testados pelo teste t a $5 \%$.

\section{RESULTADOS E DISCUSSÃO}

A análise da variância demonstrou que, nas avaliações de controle de Euphorbia heterophylla, houve interação significativa entre as doses de glyphosate e o intervalo entre a simulação de chuva e a aplicação do herbicida aos 7,34 e 41 dias após aplicação (DAA), indicando que o intervalo de chuva antes da aplicação do herbicida influenciou o seu desempenho. Aos 20 (DAA), houve efeito de dose e de intervalo, sem relação de dependência entre eles. 
Para cada período de simulação de chuva anterior à aplicação do herbicida, definiu-se o intervalo em horas; para cada intervalo, estabeleceu-se correspondência com o teor de água no solo e o potencial mátrico de água no solo no momento da aplicação (Tabela 2). O potencial mátrico de água no solo representa a energia com que a água se encontra nele retida e comanda todos os processos de transporte de água no sistema solo-planta-atmosfera. Nos intervalos de 48 e 72 horas, o solo apresentava umidade baixa no momento da aplicação de glyphosate, o que corresponde ao potencial de água no solo abaixo do Ponto de Murcha Permanente (PMP) (Tabela 2). Nesse ponto, o conteúdo de água no solo fica retido a um potencial tão elevado, que a maioria das plantas não consegue extrair água dele e entra em PMP. Embora o teor de água estivesse abaixo do PMP, as plantas não mostravam sintomas de perda de turgor; possivelmente, elas apresentavam reservas, as quais se mantiveram sem demonstrar sinais visiveis de déficit hídrico, ou, como se trata de uma espécie invasora, voltada à sobrevivência em condições adversas, ela pode ter a capacidade de extrair moléculas de água fortemente retidas.

Aos 7 dias após aplicação (DAA), observouse tendência de que aumentos progressivos na dose do herbicida implicaram incremento na intensidade de controle de E. heterophylla, independentemente do intervalo de simulação de chuva (Figura 2a e Tabela 3). Esse mesmo comportamento também pode ser observado aos 20, 34 e 41 DAA. À medida que se aumentou a dose do herbicida, ocorreu incremento no controle, ficando entre 57,7 e $74,9 \%$ e 80,7 a $87,2 \%$ nas doses de 720 e $1.080 \mathrm{~g} \mathrm{ha}^{-1}$ de gly-

Tabela 2 - Teor de água no solo determinado no momento da aplicação do herbicida e seu respectivo potencial mátrico obtido pela equação de Genucthen (1980)

\begin{tabular}{|c|c|c|}
\hline $\begin{array}{c}\text { Intervalo } \\
\text { (horas) }\end{array}$ & $\begin{array}{c}\text { Umidade } \\
\left(\mathrm{cm}^{3} \mathrm{~cm}^{-3}\right)\end{array}$ & $\begin{array}{c}\text { Potencial } \\
(-\mathrm{KPa})\end{array}$ \\
\hline 0 & 0,23 & 16,3 \\
\hline 6 & 0,20 & 36,8 \\
\hline 12 & 0,14 & 672,3 \\
\hline 24 & 0,13 & 760,9 \\
\hline 48 & 0,09 & $<$ PMP* $^{*}$ \\
\hline 72 & 0,08 & $<$ PMP* $^{*}$ \\
\hline
\end{tabular}

* Teores de água inferiores ao ponto de murcha permanente. phosate, respectivamente, já aos 7 DAA, independentemente do intervalo. Aos 20 DAA, ocorreu incremento no percentual de controle da planta daninha, atingindo na dose de $720 \mathrm{~g} \mathrm{ha}^{-1}$ controle superior a $78 \%$ e, na dose de $1.080 \mathrm{~g}$ ha ${ }^{1}$, superior a $99 \%$ para todos os intervalos (Figura $2 \mathrm{~b}$ e Tabela 3). Nas avaliações realizadas aos 34 DAA, o controle foi superior a $82 \%$ para a dose de $720 \mathrm{~g} \mathrm{ha}^{-1}$, atingindo $100 \%$ para a dose de $1.080 \mathrm{~g} \mathrm{ha}^{-1}$ (Figura $2 \mathrm{c} \mathrm{e}$ Tabela 3). Com utilização da dose de $720 \mathrm{~g} \mathrm{ha}^{-1}$, o controle foi superior a $85 \%$ aos 41 DAA para todos os intervalos, atingindo percentuais superiores a $99,5 \%$ para a dose de $1.080 \mathrm{~g} \mathrm{ha}^{-1}$ (Figura 2d e Tabela 3).

As condições climáticas no interior da casa de vegetação desde o início do período de simulação de chuva foram: temperatura média de $34,5{ }^{\circ} \mathrm{C}$ durante o dia e $24{ }^{\circ} \mathrm{C}$ durante a noite, com umidade relativa variando de 27 a $46 \%$ durante o dia e de 46 a $58 \%$ durante a noite (Figura 1). Essas condições de temperatura alta e umidade relativa baixa causam certo grau de estresse nas plantas, fazendo com que estas se utilizem de meios para evitar a perda de água por transpiração. Rapidamente após o início da incidência dessas condições, as plantas fecham os estômatos e produzem mais cera epicuticular, a qual, segundo Heredia et al. (1998), se destaca como importante barreira à perda de água. Essa alta produção de cera tem sido documentada por vários autores como sendo também a principal barreira à penetração de herbicidas hidrofilicos. Ferreira et al. (2003) relataram como possiveis principais barreiras foliares à penetração de agroquímicos em Euphorbia heterophylla o alto teor de cera epicuticular, a elevada densidade de laticíferos e a grande espessura da cutícula da face adaxial.

Embora a planta daninha em estudo apresentasse características não-favoráveis à penetração de herbicidas hidrofilicos, possivelmente potencializadas pelas condições climáticas desfavoráveis dentro da casa de vegetação, verificou-se que a partir da dose de $720 \mathrm{~g} \mathrm{ha}^{-1}$ de glyphosate aos 41 DAA o controle de $E$. heterophylla foi superior a $85,8 \%$, chegando a atingir 99,5\% na dose de $1.080 \mathrm{~g} \mathrm{ha}^{-1}$ (Figura 2), independentemente do intervalo entre a última irrigação e a aplicação do herbicida. Chachalis et al. (2001) constataram 

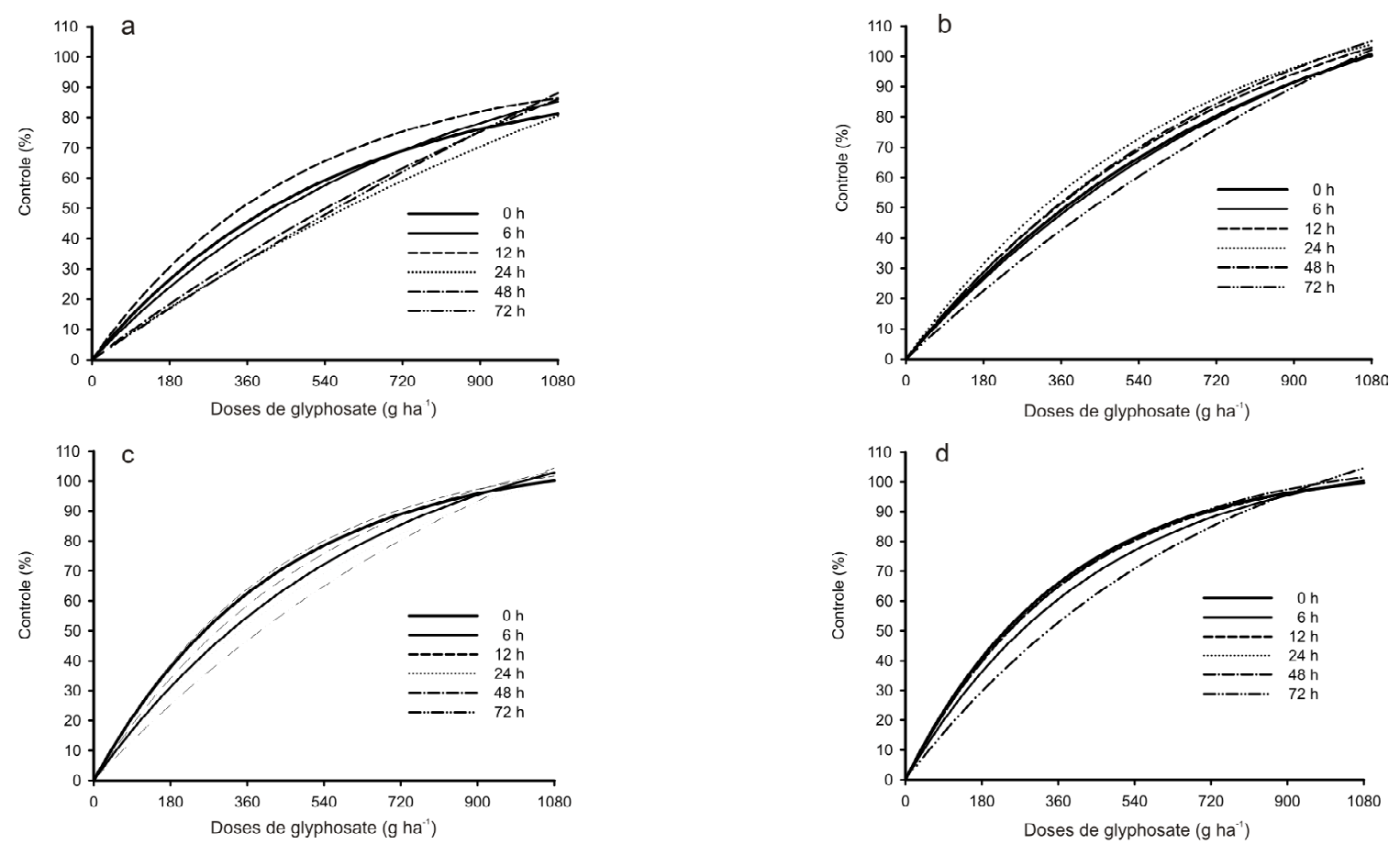

Figura 2 - Controle de Euphorbia heterophylla em seis intervalos (horas) entre a última irrigação e a aplicação de diferentes doses de glyphosate, aos 7 (a), 20 (b), 34 (c) e 41 (d) dias após pulverização do herbicida. Capão do Leão, RS.

controle de 54 e $69 \%$ para Brunnichia ovata e 62 e 78\% para Campsis radicans com a pulverização de glyphosate nas doses de 1,1 e $3,3 \mathrm{~kg} \mathrm{ha}^{-1}$, respectivamente. A baixa eficácia de controle do glyphosate em Brunnichia ovata foi atribuída pelos autores à maior natureza hidrofóbica da cera epicuticular de B. ovata, quando comparado com $C$. radicans.

Aos 7 DAA de $180 \mathrm{~g} \mathrm{ha}^{-1}$ de glyphosate, verificou-se controle de $24,0 \%$ da planta daninha no intervalo de $0 \mathrm{~h}$ (Figura $3 \mathrm{a}$ e Tabela 4), correspondente a $26,0 \%$ de controle quando o solo apresentava teor de água de $0,23 \mathrm{~cm}^{3} \mathrm{~cm}^{-3}$ (Figura 4a e Tabela 5). Com o aumento do intervalo para 72 horas, o controle de E. heterophylla decresceu para $3,5 \%$, correspondente a $7,5 \%$ quando o solo apresentava teor de água de $0,08 \mathrm{~cm}^{3} \mathrm{~cm}^{-3}$. No entanto, para a dose de $360 \mathrm{~g} \mathrm{ha}^{-1}$ o controle aumentou, atingindo $45,5 \%$ no intervalo de $0 \mathrm{~h}$ (Figura $3 \mathrm{~b} \mathrm{e}$ Tabela 4) e $45,2 \%$ para teor de água de $0,23 \mathrm{~cm}^{3} \mathrm{~cm}^{-3}$ (Figura 4a e Tabela 4). Na situação de acréscimo de intervalo, ou seja, 72 h, o controle reduziu para $23,7 \%$, o que corresponde a $27,8 \%$ de controle quando o solo apresentava teor de água de $0,08 \mathrm{~cm}^{3} \mathrm{~cm}^{-3}$.
O controle da planta daninha aumentou gradualmente com o passar das avaliações, atingindo aos 41 DAA 43,0 e 46,5\% para o intervalo de $0 \mathrm{~h}$ (Figura $3 \mathrm{~d}$ e Tabela 5 ) e teor de água no solo de $0,23 \mathrm{~cm}^{3} \mathrm{~cm}^{-3}$ (Figura $4 \mathrm{~d} \mathrm{e}$ Tabela 5) na dose de $180 \mathrm{~g} \mathrm{ha}^{-1}$, respectivamente, ao passo que, para a dose de $360 \mathrm{~g} \mathrm{ha}^{-1}$, os valores foram de 57,3 e $58,2 \%$ de controle. Na dose de $180 \mathrm{~g} \mathrm{ha}^{-1}$ o controle foi de $23,6 \%$ para o intervalo de $72 \mathrm{~h}$, correspondente a $27,4 \%$ quando o teor de água no solo era de $0,08 \mathrm{~cm}^{3} \mathrm{~cm}^{-3}$. No caso da dose de $360 \mathrm{~g} \mathrm{ha}^{-1}$, o aumento do intervalo também influenciou o controle, atingindo 48,9\% no intervalo de $72 \mathrm{~h}$, correspondendo a $51,1 \%$ para teor de água no solo de $0,08 \mathrm{~cm}^{3} \mathrm{~cm}^{-3}$.

Quando o glyphosate foi aplicado em intervalos maiores após a simulação da última irrigação ou em solo com baixa umidade e, conseqüentemente, potencial de retenção de água no solo mais negativo, ocorreu redução de sua eficiência. Seguindo esse raciocínio, quando o herbicida glyphosate é aplicado sobre plantas não-estressadas, ou seja, a intervalos mais próximos da chuva simulada, observou-se maior eficiência no controle de E. heterophylla. 
Tabela 3 - Equações das curvas de regressão relacionando controle de Euphorbia heterophylla em função da dose de glyphosate $\left(\mathrm{g} \mathrm{ha}^{-1}\right)$ em seis intervalos entre a última irrigação e a aplicação, aos 7, 20, 34 e 41 dias após pulverização do herbicida

\begin{tabular}{|c|c|c|}
\hline Intervalo $(\mathrm{h})$ & Equação & $\mathrm{R}^{2}(\%)$ \\
\hline \multicolumn{3}{|c|}{7 dias } \\
\hline 0 & $\hat{\mathrm{y}}=94,2088 *(1-\exp (-0,0018 * x))$ & 99 \\
\hline 6 & $\hat{\mathrm{y}}=111,0953 *(1-\exp (-0,0014 * x))$ & 99 \\
\hline 12 & $\hat{\mathrm{y}}=96,0566 *(1-\exp (-0,0021 * x))$ & 98 \\
\hline 24 & $\hat{\mathrm{y}}=172,0265 *(1-\exp (-0,0006 * x))$ & 99 \\
\hline 48 & $\hat{\mathrm{y}}=182,8690 *(1-\exp (-0,0006 * x))$ & 95 \\
\hline 72 & $\hat{\mathrm{y}}=297,2531 *(1-\exp (-0,0003 * x))$ & 91 \\
\hline \multicolumn{3}{|c|}{20 dias } \\
\hline 0 & $\hat{\mathrm{y}}=135,0358 *(1-\exp (-0,0013 * x))$ & 98 \\
\hline 6 & $\hat{\mathrm{y}}=142,8445 *(1-\exp (-0,0011 * \mathrm{x}))$ & 99 \\
\hline 12 & $\hat{\mathrm{y}}=135,1222 *(1-\exp (-0,0013 * \mathrm{x}))$ & 97 \\
\hline 24 & $\hat{\mathrm{y}}=127,0237 *(1-\exp (-0,0016 * x))$ & 98 \\
\hline 48 & $\hat{\mathrm{y}}=140,5908 *(1-\exp (-0,0013 * x))$ & 97 \\
\hline 72 & $\hat{\mathrm{y}}=196,5539 *(1-\exp (-0,0007 * x))$ & 95 \\
\hline \multicolumn{3}{|c|}{34 dias } \\
\hline 0 & $\hat{\mathrm{y}}=108,4492 *(1-\exp (-0,0024 * \mathrm{x}))$ & 97 \\
\hline 6 & $\hat{\mathrm{y}}=125,3127 *(1-\exp (-0,0016 * \mathrm{x}))$ & 97 \\
\hline 12 & $\hat{\mathrm{y}}=109,7036 *(1-\exp (-0,0024 * x))$ & 99 \\
\hline 24 & $\hat{\mathrm{y}}=125,3981 *(1-\exp (-0,0015 * x))$ & 99 \\
\hline 48 & $\hat{\mathrm{y}}=119,1048 *(1-\exp (-0,0019 * x))$ & 99 \\
\hline 72 & $\hat{\mathrm{y}}=165,3873 *(1-\exp (-0,0009 * x))$ & 95 \\
\hline \multicolumn{3}{|c|}{41 dias } \\
\hline 0 & $\hat{\mathrm{y}}=105,1106 *(1-\exp (-0,0027 * x))$ & 97 \\
\hline 6 & $\hat{\mathrm{y}}=110,7019 *(1-\exp (-0,0022 * \mathrm{x}))$ & 99 \\
\hline 12 & $\hat{\mathrm{y}}=106,2193 *(1-\exp (-0,0026 * x))$ & 99 \\
\hline 24 & $\hat{\mathrm{y}}=105,6021 *(1-\exp (-0,0027 * x))$ & 99 \\
\hline 48 & $\hat{\mathrm{y}}=108,8449 *(1-\exp (-0,0025 * x))$ & 98 \\
\hline 72 & $\hat{\mathrm{y}}=135,0701 *(1-\exp (-0,0014 * x))$ & 97 \\
\hline
\end{tabular}

Isso, em parte, pode ser devido à maior hidratação da cutícula da planta, o que possibilitou aumento na absorção do herbicida. A redução na absorção de glyphosate por plantas estressadas tem sido atribuída à menor hidratação da cutícula (McAllister \& Haderlie, 1985). O
Tabela 4 - Equações das curvas de regressão relacionando controle de Euphorbia heterophylla aos 7, 20, 34 e 41 dias após aplicação de diferentes doses de glyphosate, em função do intervalo entre a última irrigação e a pulverização do herbicida

\begin{tabular}{|c|c|c|}
\hline Dose $\left(\mathrm{g} \mathrm{ha}^{-1}\right)$ & Equação & $\mathrm{R}^{2}(\%)$ \\
\hline \multicolumn{3}{|c|}{7 dias } \\
\hline 0 & $\overline{\mathrm{y}}=0$ & - \\
\hline 180 & $\hat{\mathrm{y}}=24,0357+(-0,2853 * \mathrm{x})$ & 94 \\
\hline 360 & $\hat{\mathrm{y}}=45,5380+(-0,3039 * x)$ & 78 \\
\hline 720 & $\overline{\mathrm{y}}=73$ & - \\
\hline 1.080 & $\overline{\mathrm{y}}=81$ & - \\
\hline \multicolumn{3}{|c|}{20 dias } \\
\hline 0 & $\overline{\mathrm{y}}=0$ & - \\
\hline 180 & $\hat{\mathrm{y}}=29,4589+\left(-0,1713^{*} \mathrm{x}\right)$ & 97 \\
\hline 360 & $\hat{\mathrm{y}}=47,1039+\left(-0,1643^{*} \mathrm{x}\right)$ & 62 \\
\hline 720 & $\overline{\mathrm{y}}=92$ & - \\
\hline 1.080 & $\overline{\mathrm{y}}=98$ & - \\
\hline \multicolumn{3}{|c|}{34 dias } \\
\hline 0 & $\overline{\mathrm{y}}=0$ & - \\
\hline 180 & $\hat{\mathrm{y}}=38,4884+(-0,2403 * x)$ & 73 \\
\hline 360 & $\hat{\mathrm{y}}=52,3364+\left(-0,1915^{*} \mathrm{x}\right)$ & 97 \\
\hline 720 & $\bar{y}=94$ & - \\
\hline 1.080 & $\bar{y}=99$ & - \\
\hline \multicolumn{3}{|c|}{41 dias } \\
\hline 0 & $\overline{\mathrm{y}}=0$ & - \\
\hline 180 & $\hat{\mathrm{y}}=42,9085+\left(-0,2682^{*} \mathrm{x}\right)$ & 81 \\
\hline 360 & $\hat{\mathrm{y}}=57,3271+\left(-0,1171^{*} \mathrm{x}\right)$ & 90 \\
\hline 720 & $\overline{\mathrm{y}}=96$ & - \\
\hline 1.080 & $\overline{\mathrm{y}}=99$ & - \\
\hline
\end{tabular}

glyphosate é um herbicida aniônico e tem alta solubilidade em água. Segundo Silva et al. (2000), no caso dos herbicidas hidrofilicos (baixo kow, como o glyphosate), essa água de hidratação da cutícula é a sua principal rota de penetração.

O maior controle observado em intervalos mais próximos entre a chuva simulada e a aplicação do herbicida coincide com a menor massa seca de raízes e da parte aérea. A redução na atividade do glyphosate em plantas em déficit hídrico está relacionada à menor 

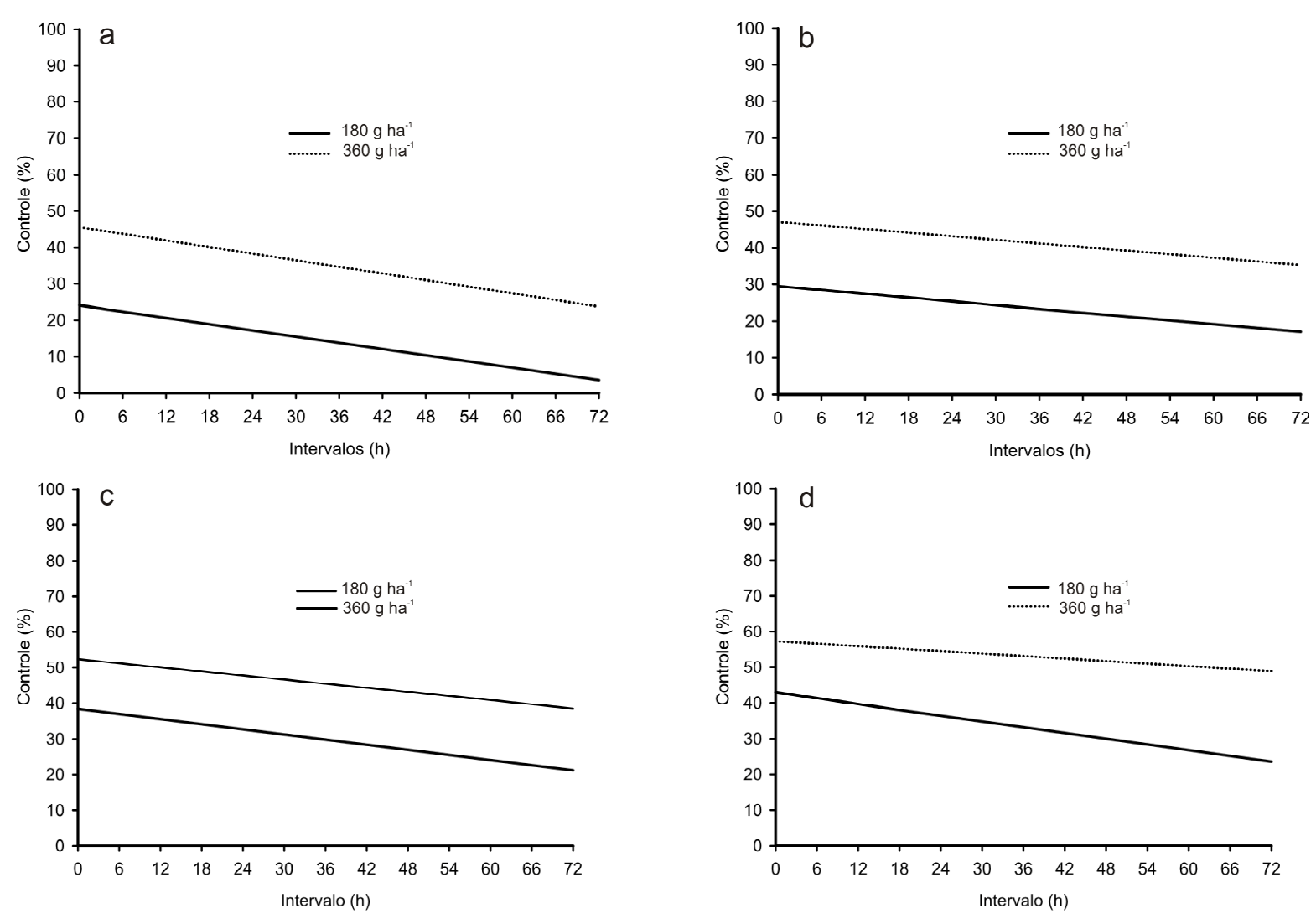

Figura 3 - Controle de Euphorbia heterophylla aos 7 (a), 20 (b), 34 (c) e 41 (d) dias após aplicação de duas doses de glyphosate, em função do intervalo entre a última irrigação e a pulverização do herbicida. Capão do Leão, RS.

absorção do herbicida e subseqüente translocação para os meristemas, o que está associado com o decréscimo do transporte de fotoassimilados no floema devido à diminuição da atividade metabólica (Ahmadi et al., 1980; McWhorter et al., 1980; Waldecker \& Wyse, 1985). Plantas de Asclepias syriaca mantidas em solo com $25 \%$ de umidade absorveram $44 \%$ do ${ }^{14} \mathrm{C}$-glyphosate aplicado e translocaram $20 \%$ do absorvido, enquanto plantas que cresceram em solo com $13 \%$ de umidade absorveram 29\% e translocaram 7\% (Waldecker \& Wyse, 1985).

Quanto à avaliação de massa seca de raízes (MSR), observou-se interação significativa entre dose e intervalo. Entretanto, para massa seca da parte aérea (MSPA) constatouse apenas efeito significativo de dose. Quando a variável avaliada foi efeito de doses do herbicida glyphosate sobre a massa seca de raízes, observou-se que ocorreu redução com o aumento das doses do herbicida. Com a pulverização de 180, 360, 720 e $1.080 \mathrm{~g} \mathrm{ha}^{-1}$ de glyphosate, ocorreu redução de 26,1 ; 65,7; 87,4 ; e $90,1 \%$ na massa seca de raízes, respectivamente, em relação à testemunha (Figura 5 e Tabela 6).

A massa seca da parte aérea apresentou o mesmo efeito observado nas raizes, ocorrendo para as doses de 180, 360, 720 e $1.080 \mathrm{~g} \mathrm{ha}^{-1}$ de glyphosate redução na MSPA de 40,7; 69,3; 90,6; e 93,2\%, respectivamente, em relação à testemunha (Figura 5 e Tabela 6).

O aumento do intervalo entre a simulação de chuva (Figura 6 e Tabela 7) e a pulverização do herbicida ou entre esta e a redução do teor de água no solo (Figura 7 e Tabela 8) no momento da aplicação do glyphosate promoveu incremento na massa seca de raízes e da parte aérea.

No intervalo de $0 \mathrm{~h}$ a MSR foi de $0,74 \mathrm{~g}$ (Figura 6 e Tabela 7), correspondente a 0,67 g para teor de água no solo de $0,23 \mathrm{~cm}^{3} \mathrm{~cm}^{-3}$ 
Tabela 5 - Equações das curvas de regressão relacionando controle de Euphorbia heterophylla aos 7, 20, 34 e 41 dias após a aplicação de diferentes doses de glyphosate, em função do teor de água no solo no momento da pulverização do herbicida

\begin{tabular}{|c|c|c|}
\hline Dose $\left(\mathrm{g} \mathrm{ha}^{-1}\right)$ & Equação & $\mathrm{R}^{2}(\%)$ \\
\hline \multicolumn{3}{|c|}{7 dias } \\
\hline 0 & $\bar{y}=0$ & - \\
\hline 180 & $\hat{\mathrm{y}}=-2,3493+(123,0986 * x)$ & 93 \\
\hline 360 & $\hat{\mathrm{y}}=18,5052+(116,0563 * x)$ & 88 \\
\hline 720 & $\bar{y}=73$ & - \\
\hline 1.080 & $\bar{y}=81$ & - \\
\hline \multicolumn{3}{|c|}{20 dias } \\
\hline 0 & $\bar{y}=0$ & - \\
\hline 180 & $\hat{\mathrm{y}}=13,9653+(73,8028 * x)$ & 87 \\
\hline 360 & $\hat{\mathrm{y}}=25,9512+\left(111,8310^{*} \mathrm{x}\right)$ & 87 \\
\hline 720 & $\bar{y}=92$ & - \\
\hline 1.080 & $\bar{y}=98$ & - \\
\hline \multicolumn{3}{|c|}{34 dias } \\
\hline 0 & $\bar{y}=0$ & - \\
\hline 180 & $\hat{\mathrm{y}}=15,4986+\left(113,8028^{*} \mathrm{x}\right)$ & 75 \\
\hline 360 & $\hat{\mathrm{y}}=35,7709+\left(78,5915^{*} \mathrm{x}\right)$ & 76 \\
\hline 720 & $\bar{y}=94$ & - \\
\hline 1.080 & $\bar{y}=99$ & - \\
\hline \multicolumn{3}{|c|}{41 dias } \\
\hline 0 & $\bar{y}=0$ & - \\
\hline 180 & $\hat{\mathrm{y}}=17,1230+(127,8873 * x)$ & 85 \\
\hline 360 & $\hat{\mathrm{y}}=47,2638+\left(47,6056^{*} \mathrm{x}\right)$ & 69 \\
\hline 720 & $\bar{y}=96$ & - \\
\hline 1.080 & $\bar{y}=99$ & - \\
\hline
\end{tabular}

(Figura 7 e Tabela 8), para a dose de $180 \mathrm{~g} \mathrm{ha}^{-1}$ de glyphosate. Nesta mesma dose, a MSR aumentou para $1,02 \mathrm{~g}$ no intervalo de $72 \mathrm{~h}$, correspondente a $0,98 \mathrm{~g}$ para teor de água no solo de $0,08 \mathrm{~cm}^{3} \mathrm{~cm}^{-3}$. Para a dose de $360 \mathrm{~g} \mathrm{ha}^{-1} \mathrm{de}$ glyphosate, a MSR foi de 0,37 g no intervalo de $0 \mathrm{~h}$, correspondente a $0,34 \mathrm{~g}$ para teor de água no solo de $0,23 \mathrm{~cm}^{3} \mathrm{~cm}^{-3}$. No entanto, para o intervalo de $72 \mathrm{~h}$, a MSR aumentou para 0,56 g,
Tabela 6 - Equações das curvas de regressão relacionando altura de plantas de Euphorbia heterophylla em função da dose de glyphosate (g e.a. ha ${ }^{-1}$ em seis intervalos entre a última irrigação e a aplicação, aos 7, 20, 34 e 41 dias após a pulverização do herbicida

\begin{tabular}{|c|c|c|}
\hline Intervalo (h) & Equação & $\mathrm{R}^{2}(\%)$ \\
\hline \multicolumn{3}{|c|}{ MSR } \\
\hline 0 & $\hat{\mathrm{y}}=1,3228 *(1-\exp (-0,0034 * x))$ & 98 \\
\hline 6 & $\hat{\mathrm{y}}=1,0713 *(1-\exp (-0,0028 * x))$ & 97 \\
\hline 12 & $\hat{\mathrm{y}}=1,2061 *(1-\exp (-0,0028 * x))$ & 96 \\
\hline 24 & $\hat{\mathrm{y}}=1,0829 *\left(1-\exp \left(-0,0026^{*} \mathrm{x}\right)\right)$ & 97 \\
\hline 48 & $\hat{\mathrm{y}}=1,0972 *(1-\exp (-0,0023 * x))$ & 87 \\
\hline 72 & $\hat{\mathrm{y}}=1,1404 *(1-\exp (-0,0020 * x))$ & 92 \\
\hline \multicolumn{3}{|c|}{ MSPA } \\
\hline 0 & $\hat{\mathrm{y}}=5,2810 *(1-\exp (-0,0036 * x))$ & 98 \\
\hline 6 & $\hat{\mathrm{y}}=5,0653 *\left(1-\exp \left(-0,0033^{*} \mathrm{x}\right)\right)$ & 99 \\
\hline 12 & $\hat{\mathrm{y}}=5,8306 *(1-\exp (-0,0032 * x))$ & 99 \\
\hline 24 & $\hat{\mathrm{y}}=5,9775 *(1-\exp (-0,0034 * x))$ & 99 \\
\hline 48 & $\hat{\mathrm{y}}=5,3650 *(1-\exp (-0,0029 * x))$ & 97 \\
\hline 72 & $\hat{\mathrm{y}}=5,2563 *(1-\exp (-0,0025 * x))$ & 98 \\
\hline
\end{tabular}

o que equivale a $0,51 \mathrm{~g}$ para teor de água no solo de $0,08 \mathrm{~cm}^{3} \mathrm{~cm}^{-3}$.

Em relação à MSPA, com a pulverização de $180 \mathrm{~g} \mathrm{ha}^{-1}$ de glyphosate no intervalo de $0 \mathrm{~h}$, ela foi de 2,75 g (Figura 6 e Tabela 7), correspondente a 2,45 g para teor de água no solo de $0,23 \mathrm{~cm}^{3} \mathrm{~cm}^{-3}$ (Figura 7 e Tabela 8). Com o aumento do intervalo entre a simulação de chuva e a pulverização do herbicida, a MSPA aumentou, atingindo $3,84 \mathrm{~g}$ no intervalo de $72 \mathrm{~h}$, o que corresponde a 3,70 g para teor de água no solo de $0,08 \mathrm{~cm}^{3} \mathrm{~cm}^{-3}$. Com o aumento da dose de aplicação do herbicida (360 $\mathrm{g} \mathrm{ha}^{-1}$ ), ocorreu redução na MSPA em relação à dose de $180 \mathrm{~g} \mathrm{ha}^{-1}$. Quanto à dose de $360 \mathrm{~g} \mathrm{ha}^{-1}$, a MSPA foi de $1,58 \mathrm{~g}$ no intervalo de $0 \mathrm{~h}$ (Figura $6 \mathrm{e}$ Tabela 7), correspondente a $1,54 \mathrm{~g}$ para teor de água no solo de $0,23 \mathrm{~cm}^{3} \mathrm{~cm}^{-3}$ (Figura $7 \mathrm{e}$ Tabela 8). Com o aumento do intervalo houve redução na MSPA, atingindo no intervalo de $72 \mathrm{~h} 2,00 \mathrm{~g}$, equivalente a $1,93 \mathrm{~g}$ para teor de água no solo de $0,08 \mathrm{~cm}^{3} \mathrm{~cm}^{-3}$. Dickson et al. (1990) relataram que, 32 dias após pulverização de $0,5 \mathrm{~kg} \mathrm{ha}^{-1}$ de fluazifop-p-butyl, a massa seca da parte aérea foi maior em plantas em estresse hídrico $(0,64 \mathrm{~g})$ do que em plantas não-estressadas $(0,14 \mathrm{~g})$. 

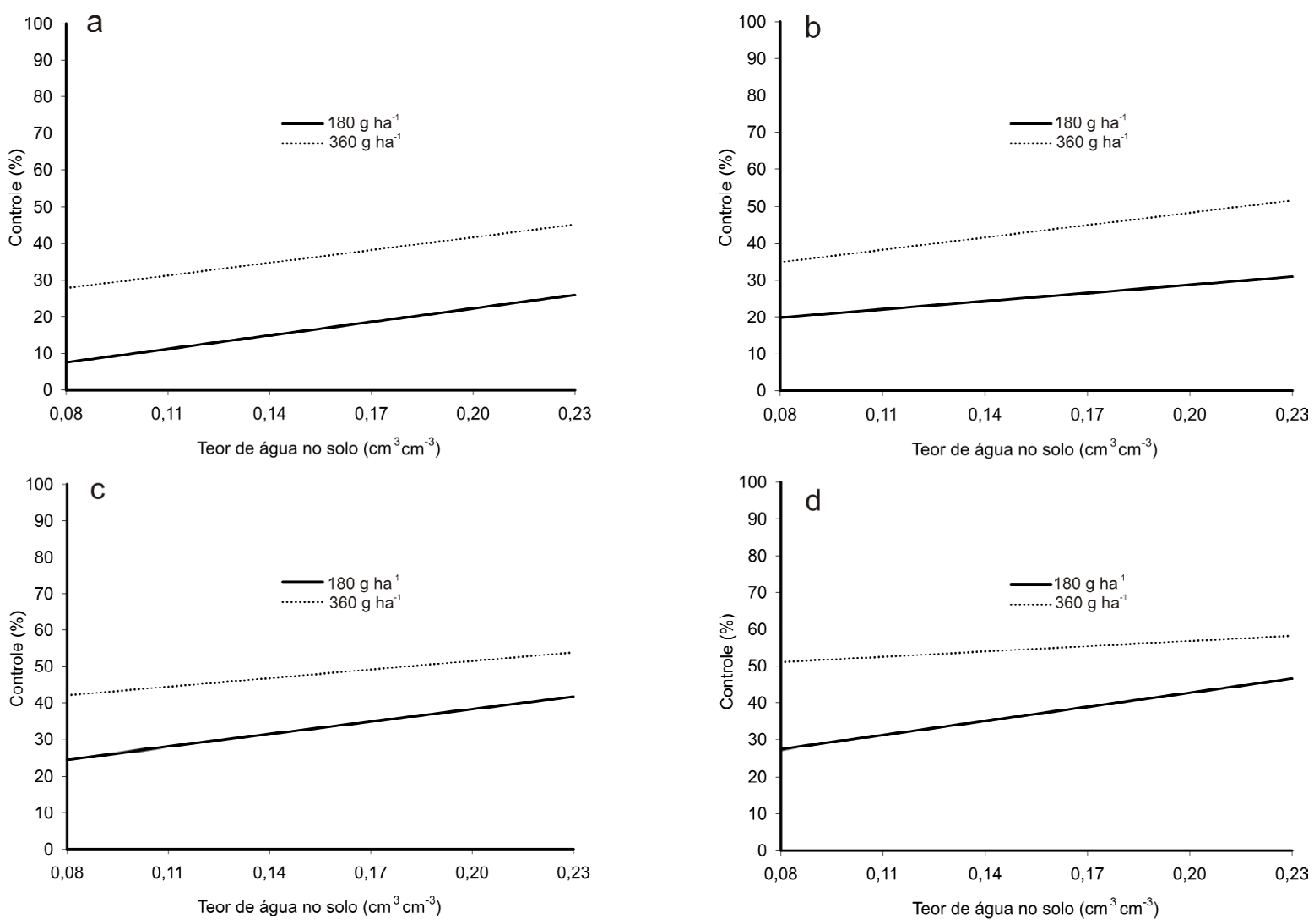

Figura 4 - Controle de Euphorbia heterophylla aos 7 (a), 20 (b), 34 (c) e 41 (d) dias após aplicação de duas doses de glyphosate, em função do teor de água no solo no momento da pulverização do herbicida. Capão do Leão, RS.
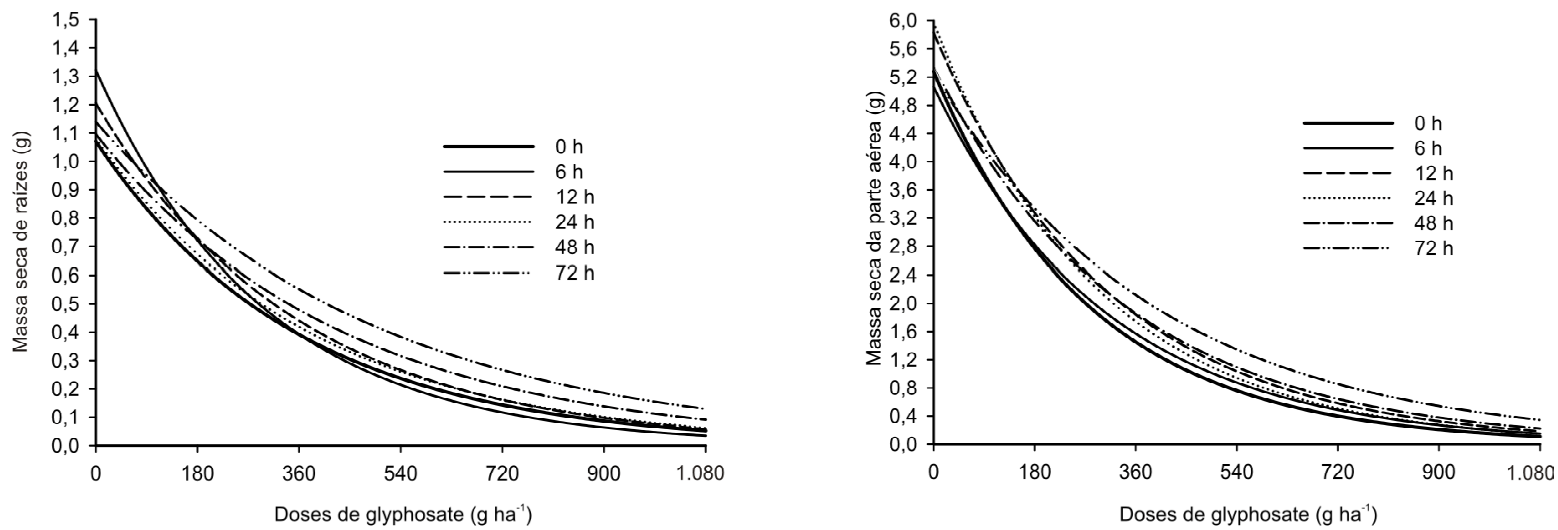

Figura 5 - Massa seca das raízes e da parte aérea de Euphorbia heterophylla em seis intervalos (horas) entre a última irrigação e a aplicação de diferentes doses de glyphosate, aos 41 dias após pulverização do herbicida. Capão do Leão, RS. 

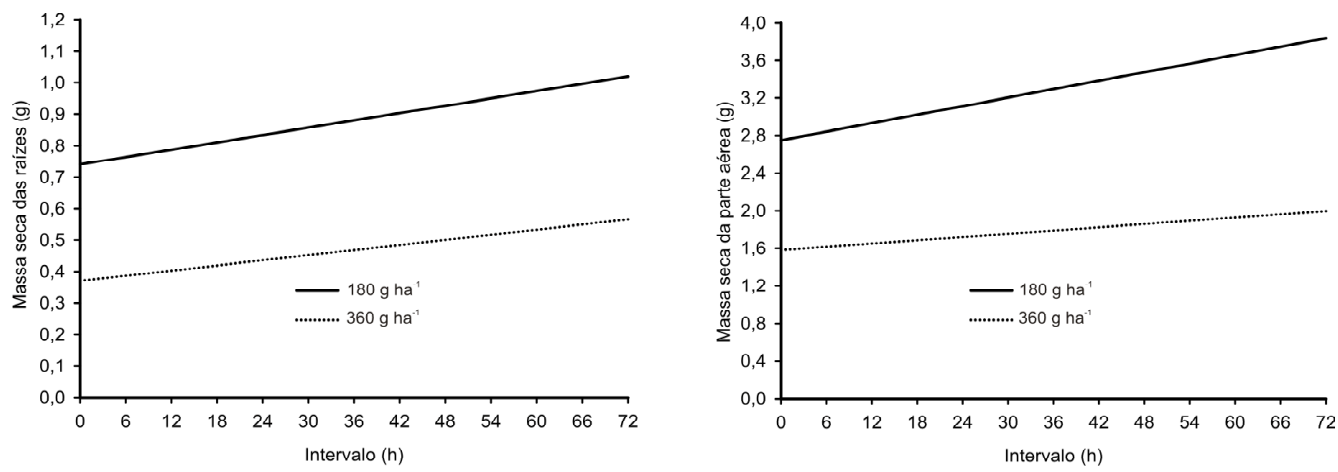

Figura 6 - Massa seca de raízes e da parte aérea de Euphorbia heterophylla aos 41 dias após aplicação de duas doses de glyphosate, em função do intervalo entre a última irrigação e a pulverização do herbicida. Capão do Leão, RS.
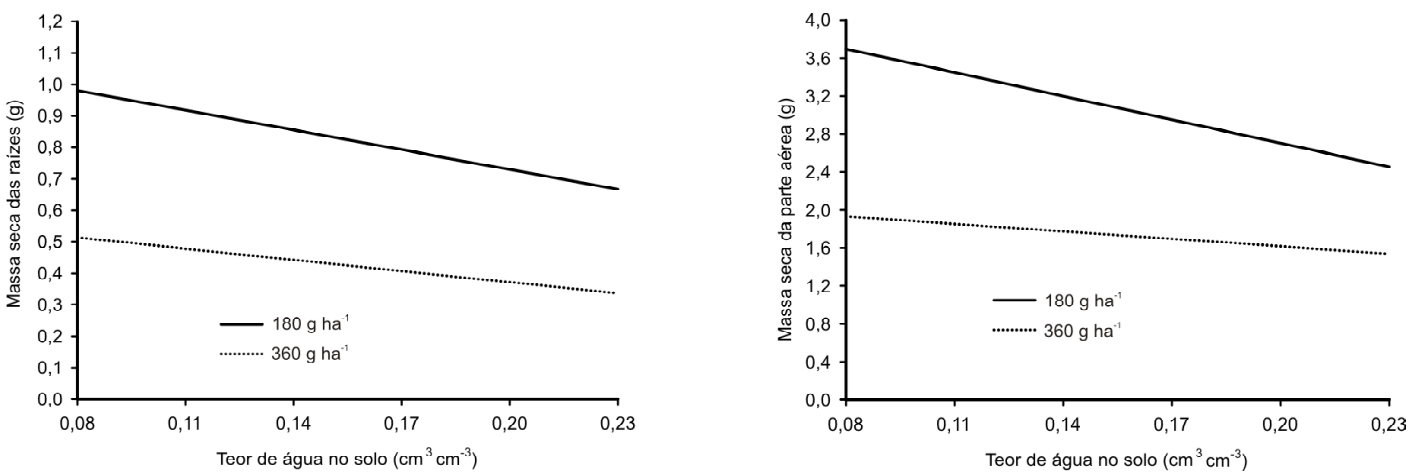

Figura 7 - Massa seca de raízes e da parte aérea de Euphorbia heterophylla aos 41 dias após aplicação de duas doses de glyphosate, em função do teor de água no solo no momento da pulverização do herbicida. Capão do Leão, RS.

Tabela 7 - Equações das curvas de regressão relacionando massa seca das raízes (MSR) e da parte aérea (MSPA) de Euphorbia heterophylla aos 41 dias após aplicação de diferentes doses de glyphosate, em função de intervalo entre a última irrigação e a pulverização do herbicida

\begin{tabular}{|c|c|c|}
\hline Dose $\left(\mathrm{g} \mathrm{ha}^{-1}\right)$ & Equação & $\mathrm{R}^{2}(\%)$ \\
\hline \multicolumn{3}{|c|}{ MSR } \\
\hline 0 & $\overline{\mathrm{y}}=1,1$ & - \\
\hline 180 & $\hat{\mathrm{y}}=0,7401+\left(0,0039^{*} \mathrm{x}\right)$ & 73 \\
\hline 360 & $\hat{\mathrm{y}}=0,3701+\left(0,0027^{*} \mathrm{x}\right)$ & 77 \\
\hline 720 & $\overline{\mathrm{y}}=0,14$ & - \\
\hline 1.080 & $\overline{\mathrm{y}}=0,11$ & - \\
\hline \multicolumn{3}{|c|}{$\mathrm{MSPA}$} \\
\hline 0 & $\overline{\mathrm{y}}=5,43$ & - \\
\hline 180 & $\hat{\mathrm{y}}=2,7504+\left(0,0151^{*} \mathrm{x}\right)$ & 71 \\
\hline 360 & $\hat{\mathrm{y}}=1,5809+\left(0,0058^{*} \mathrm{x}\right)$ & 72 \\
\hline 720 & $\overline{\mathrm{y}}=0,51$ & - \\
\hline 1.080 & $\overline{\mathrm{y}}=0,37$ & - \\
\hline
\end{tabular}

Tabela 8 - Equações das curvas de regressão relacionando massa seca das raízes (MSR) e da parte aérea (MSPA) de Euphorbia heterophylla aos 41 dias após aplicação de diferentes doses de glyphosate, em função do teor de água no solo no momento da pulverização do herbicida

\begin{tabular}{|c|c|c|}
\hline Dose $\left(\mathrm{g} \mathrm{ha}^{-1}\right)$ & Equação & $\mathrm{R}^{2}(\%)$ \\
\hline \multicolumn{3}{|c|}{ MSR } \\
\hline 0 & $\overline{\mathrm{y}}=1,1$ & - \\
\hline 180 & $\hat{\mathrm{y}}=1,1485+\left(-2,0930^{*} \mathrm{x}\right)$ & 98 \\
\hline 360 & $\hat{\mathrm{y}}=0,6082+\left(-1,1831^{*} \mathrm{x}\right)$ & 67 \\
\hline 720 & $\overline{\mathrm{y}}=0,14$ & - \\
\hline 1.080 & $\overline{\mathrm{y}}=0,11$ & - \\
\hline & $\mathrm{MSPA}$ & - \\
\hline 0 & $\overline{\mathrm{y}}=5,43$ & 98 \\
\hline 180 & $\hat{\mathrm{y}}=4,3588+(-8,2789 * \mathrm{x})$ & 63 \\
\hline 360 & $\hat{\mathrm{y}}=2,1399+(-2,6197 * \mathrm{x})$ & - \\
\hline 720 & $\overline{\mathrm{y}}=0,51$ & - \\
\hline 1.080 & $\overline{\mathrm{y}}=0,37$ & \\
\hline
\end{tabular}




\section{LITERATURA CITADA}

AHMADI, M. S.; HADERLIE, L. C.; WICKS, G. A. Effects of growth stage and water stress on barnyardgrass (Echinochlo a crus-galli) control and on glyphosate absorption and translocation. Weed Sci., v. 28, p. 277-282, 1980.

ANDERSON, D. M. et al. The influence of temperature and humidity on the efficacy of glufosinato ammonium. Weed Res., v. 33, p. 139-147, 1993.

BASLER, E.; TODD, G. W.; MEYER, R. E. Effects of moisture stress on absorption, translocation and distribution of 2,4-dichlorophenoxyacet acid in bean plants. Plant Physiol., v. 36, p. 573-579, 1961.

BOYDSTON, R. A. Soil water content affects the activity of four herbicides on green foxtail. Weed Sci., v. 38, p. 578$582,1990$.

BRIDGES, D. C.; BRECKE, B. J.; BARBOUR, J. C. Wild poinsettia (Euphorbia heterophylla) interference with peanut (Arachis hypogaea). Weed Sci., v. 40, p. 37-42, 1992.

CASELEY, J. C.; COUPLAND, D. Environmental and plant factors affecting glyphosate uptake, movement and activity, In: GROSSBARD, E.; ATKINSON, D. (Eds.). The herbicide glyphosate London:Marcel Dekker, 1985. p.92-123.

CHACHALIS, D.; REDDY, K. N.; ELMORE, C. D. Characterization of leaf surface, wax composition, and control of redvine and trumpetcreeper with glyphosate. Weed Sci., v. 49, p. 156-163, 2001.

DE RUITER, H. et al. Influence of surfactants and plant species on leaf retention of spray solutions. Weed Sci., v. 38, p. $567-572,1990$.

DICKSON, R. L. et al. Effect of water stress, nitrogen, and gibberellic acid on fluazifop and glyphosate activity on oats (Avena sativa). Weed Sci., v. 38, p. 54-61, 1990.

\section{EMPRESA BRASILEIRA DE PESQUISA}

AGROPECUÁRIA - EMBRAPA. Centro Nacional de Pesquisa de Solos. Manual de métodos de análise de solo. 2.ed. Rio de Janeiro: 1997. 212 p.

FENG, P. C. C. et al. Droplet size affects glyphosate retention, absorption and translocation in corn. Weed Sci., v. 51, p. 443-448, 2003.

FERREIRA, E. A. et al. Estudos anatômicos de folhas de espécies de plantas daninhas de grande ocorrência no Brasil. IV - Amaranthus deflexus, Amaranthus spinosus, Alternanthera tenella e Euphorbia heterophylla. Planta Daninha, v. 21, p. 263-271, 2003.
HEREDIA, A. et al. La cutícula vegetal: estructura y funciones. Ecologia, v. 12, p. 293-305, 1998.

HESS, F. D. Mechanism of action of inhibitors of amino acid biosynthesis. In: Herbicide action: an intensive course on the activity, selectivity, behavior, and fate of herbicides in plants and soil. West Lafayette: Purdue University, 1994. p. 344-365.

KIDDER, D. W.; BEHRENS, R. Control of plant water potential in water stress studies. Weed Sci., v. 39, p. 91-96, 1991.

KIDDER, D. W.; BEHRENS, R. Plant response to haloxyfop as influenced by water stress. Weed Sci., v. 36, p. $305-311,1988$

KISSMANN, K. G.; GROTH, D. Plantas infestantes e nocivas. São Paulo: Basf, 1999. 978 p.

KLUTE, A. Water retention: laboratory methods. In: KLUTE, A. Methods of soil analysis . Madison: American Society of Agronomy, Soil Science Society of America, 1986. p. $635-660$

KOGAN, M.; BAYER, D. E. Herbicide uptake as influenced by plant water status. Pestic. Biochem. Physiol., v. 56, p.174-182, 1996

LEVENE, B. C.; OWEN, M. D. K. Effect of moisture stress and leaf age on bentazon absorption in common cocklebur (Xanthium strumarium) and velvetleaf (Abutilon theophrasti). Weed Sci., v. 43, p. 7-12, 1995.

MCALLISTER, R. S.; HADERLIE, L. C. Translocation of ${ }^{14} \mathrm{C}$-glyphosate and ${ }^{14} \mathrm{CO}_{2}$-labeled photoassimilates in Canada thistle (Cirsium arvense). Weed Sci., v. 33, p.153159, 1985.

MCWHORTER, C. G.; JORDAN, T. N.; WILLS, G. D. Translocation of 14C-glyphosate in soybeans (Glycine max) and johnsongrass (Sorghum halepense). Weed Sci., v. 28, p. 113-118, 1980.

MENDONÇA, G. G. Algumas características da superfície foliar de diversas plantas daninhas monocotiledôneas. 2000. 89 f. Dissertação (Mestrado em Fitotecnia) Universidade Estadual Paulista, Botucatu, 2000.

MONQUERO, P. A. et al. Caracterização da superfície foliar e das ceras epicuticulares em Commelina benghalensis, Ipomoea grandifolia e Amaranthus hybridus . Planta Daninha, v. 22, p. 203-210, 2004.

MOORE, J. D.; BANKS, P. A.; PINNEL-ALISON, C. L. Wild poinsettia (Euphorbia heterophylla) control in peanut (Arachis hypogaea). Weed Sci., v. 38, p. 536-540, 1990. 
MOOSAVI-NIA, H.; DORE, J. Factors affecting glyphosate activity in Imperata cylindrica (L.) Beau. and Cyperus rotundus L. I. Effect of soil moisture. Weed Res., v. 19, p. 137-143, 1979.

NALEWAJA, J. D.; WOZNICA, Z. Environment and chlorsulfuron phytotoxicity. Weed Sci., v. 22, p. 395-399, 1985.

PEREGOY, R. et al. Moisture stress effects on the absorption, translocation, and metabolism of haloxyfop in johnsongrass (Sorghum halepense) and large crabgrass (Digitaria sanguinalis). Weed Sci., v. 38, p. 331-337, 1990.

REDE OFICIAL DE LABORATÓRIOS DE ANÁLISE DE SOLO E DE TECIDO VEGETAL - ROLAS. Manual de adubação e calagem para os estados do Rio Grande do Sul e Santa Catarina. 10.ed. Porto Alegre: Sociedade Brasileira de Ciência do Solo, 2004. 400 p.

RODRIGUES, B. N.; ALMEIDA, F. S. Guia de herbicidas. Londrina: Edição dos Autores, 1998. 648 p.

SHARMA, S. D.; SINGH, M. Environmental factors affecting absorption and bio-efficacy of glyphosate in florida beggarweed (Desmodium tortuosum). Crop Protec., v. 20, p. 511-516, 2001.
SANTOS, J. L. et al. Influência do orvalho na eficiência do glyphosate sobre Brachiaria decumbens. Planta Daninha, v. 22, p. 285-291, 2004.

SILVA, A. A. et al. Controle de plantas daninhas. Brasília: ABEAS, 2000. p. 260.

van GENUCHTEN, M. Th. A closed form equation for predicting the hydraulic condutivity of unsaturated soils. Soil Sci. Soc. Am. J., v. 44, p. 892-898, 1990.

VICTORIA FILHO, R. Fatores que influenciam a absorção foliar dos herbicidas. Inf. Agropec., v. 11, p. 31-37, 1985.

VIDAL, R. A.; WINKLER, L. M. Resistência de plantas daninhas: seleção ou indução à mutação pelos herbicidas inibidores de acetolactato sintase (ALS). Pesticidas: R. Ecotoxicol. Meio Amb., v. 12, p. 31-42, 2002.

WALDECKER, M. A.; WYSE, D. L. Chemical and physical effects on the acumulation of glyphosate in common milkweed (Asclepias syriaca) root buds. Weed Sci., v. 33, p. 605-611, 1985.

WILLARD, T. S.; GRIFFIN, J. L. Soybean (Glycine max) yield and quality responses associated with wild poinsettia (Euphorbia heterophylla) control programs. Weed Technol., v. 7, p. 118-122, 1993. 